\begin{tabular}{ll|l} 
Case Reports in & \multicolumn{2}{c}{ Case Rep Gastroenterol 2017;11:422-427 } \\
\cline { 2 - 3 } Gastroenterology & $\begin{array}{l}\text { DOI: 10.1159/000478722 } 2017 \text { The Author(s) } \\
\text { Publisned onIne: August 4, } 2017\end{array}$ \\
\cline { 2 - 3 } & $\begin{array}{l}\text { Published by S. Karger AG, Basel } \\
\text { www.karger.com/crg }\end{array}$ \\
\cline { 2 - 3 } & $\begin{array}{l}\text { This article is licensed under the Creative Commons Attribution-NonCommercial } 4.0 \\
\text { International License (CC BY-NC) (http://www.karger.com/Services/OpenAccessLicense). } \\
\text { Usage and distribution for commercial purposes requires written permission. }\end{array}$
\end{tabular}

\title{
Resection of Locally Advanced Pancreatic Neoplasms after Neoadjuvant Chemotherapy with Nab-Paclitaxel and Gemcitabine following FOLFIRINOX Failure
}

\author{
Sarah Hahn ${ }^{a}$ Ahmet Ayav ${ }^{b}$ Anthony Lopez \\ ${ }^{a}$ Department of Gastroenterology and Hepatology and Inserm U954, Nancy \\ University Hospital, Lorraine University, Vandoeuvre-lès-Nancy, France; \\ ${ }^{b}$ Department of HPB Surgery, University Hospital Nancy Brabois, Lorraine \\ University, Vandoeuvre-lès-Nancy, France
}

\section{Keywords}

Pancreas · Drug therapy $\cdot$ Surgery $\cdot$ Tumor

\begin{abstract}
The incidence of pancreatic cancer has dramatically increased over the past years, but the prognosis has not improved. Between 30 and $40 \%$ of tumors are considered locally advanced, essentially due to vascular involvement. In recent years, new chemotherapy protocols with high response rates have been developed. FOLFIRINOX seems to be an interesting option in this situation, but hematologic toxicity could be an obstacle to its prescription. Nabpaclitaxel and gemcitabine offer significant response rates with a reasonable safety profile. We report here a single-center experience of 2 cases with a locally advanced pancreatic cancer initially considered unresectable, progressive after first-line neoadjuvant FOLFIRINOX chemotherapy, and then treated with second-line nab-paclitaxel/gemcitabine chemotherapy.

(C) 2017 The Author(s)

Published by S. Karger AG, Basel
\end{abstract}




\section{Introduction}

Pancreatic cancer is the second most frequent digestive neoplasm after colorectal cancer [1], and it is expected to be the second leading cause of cancer-related death by 2030 in the US [2]. With an overall survival of $7 \%$ at 5 years, the prognosis of pancreatic cancer has not improved over the past two decades [1]. Surgical resection is the only available potentially curative treatment, with a survival rate of $20 \%$ at 5 years, but only $15-20 \%$ of patients can benefit from it [3]. Locally advanced pancreatic cancer, either unresectable or borderline resectable, is often treated with systemic neoadjuvant chemotherapy, with the hope of surgical cure. This situation concerns between 30 and $40 \%$ of patients [1]. Due to the heterogeneity and small sample size of available retrospective series, it is difficult to recommend a specific schedule of treatment for these patients. Since 2011, chemotherapies with response rates between 20 and $30 \%$ are used in metastatic pancreatic cancer patients based on two phase III studies evaluating FOLFIRINOX [4] and nab-paclitaxel/gemcitabine [5]. We report here 2 cases with a locally advanced pancreatic cancer initially considered unresectable, progressive after first-line neoadjuvant FOLFIRINOX chemotherapy, and then treated with second-line nab-paclitaxel/gemcitabine chemotherapy.

\section{Case 1}

We present the case of a 44-year-old man with a history of glaucoma. He smoked about one pack of cigarettes a day during 25 years and stopped 1 year before his first visit at our institution. He suffered from persistent epigastric pain during 2 months that led to a gastroenterologist consultation in April 2015. Due to CA19-9 values four times the upper limit of normal, an endoscopic ultrasonography was performed, finding a mass of the pancreatic isthmus in contact with the superior mesenteric vein (SMV). The initial computed tomography (CT) scan demonstrated a mass of the pancreatic body in contact with the celiac axis (CA) and common hepatic artery (CHA) encasement $>180^{\circ}$, but without regional lymphadenopathy or distant metastasis (Fig. 1a). Given the contact with the CA and the CHA, the tumor was deemed locally advanced and nonresectable.

Based on the Eastern Cooperative Oncology Group (ECOG) status of 0, the patient began a systemic treatment with FOLFIRINOX (oxaliplatin $85 \mathrm{mg} / \mathrm{m}^{2}$ of body surface area, leucovorin $400 \mathrm{mg} / \mathrm{m}^{2}$, irinotecan $90 \mathrm{mg} / \mathrm{m}^{2}$, and 5 -fluorouracil $400 \mathrm{mg} / \mathrm{m}^{2}$ given as a bolus followed by $2,400 \mathrm{mg} / \mathrm{m}^{2}$ as a $46-\mathrm{h}$ continuous infusion, every 2 weeks, with granulocyte colony-stimulating factor support). Four cycles of chemotherapy were administered without major toxicity.

After 2 months of treatment, the patient underwent a restaging CT scan, which showed partial regression of the tumor of about $10 \%$ and a persistent contact with the CA and the CHA $>180^{\circ}$ (Fig. 1b). The tumor was still not resectable after four cycles of first-line chemotherapy, so we initiated a second line of systemic chemotherapy with a nab-paclitaxel/gemcitabine regimen. Three cycles were administered $\left(1,000 \mathrm{mg} / \mathrm{m}^{2}\right.$ of gemcitabine and $125 \mathrm{mg} / \mathrm{m}^{2}$ of nab-paclitaxel on days 1,8 , and 15 every 28 days), without major toxicity. The patient underwent a new restaging CT scan after the three cycles of chemotherapy, which showed good tumoral response, with regression of the perivascular infiltration (Fig. 1c). We also noticed a decrease in the CA19-9 level (Fig. 2). According to the tumoral response on the CT scan and the young age of the patient, he was offered surgical exploration. The latter did not find any peritoneal carcinomatosis or liver metastasis, and intraoper- 
ative frozen sections of tissue around the CHA showed no evidence of malignancy, so that a distal pancreatectomy was performed. The pathological tumor stage was ypT2N0. It was a R0 resection, with security margins $>1 \mathrm{~mm}$.

The patient received additional nab-paclitaxel/gemcitabine as adjuvant treatment for 3 months, considering efficacy of this regimen before surgery. Unfortunately, 2 months after the end of chemotherapy, CT scan showed local tumoral recurrence and signs of peritoneal carcinomatosis. At present he has been treated with palliative chemotherapy for 6 months.

\section{Case 2}

We present the case of a 62-year-old woman with a history of diabetes and arterial hypertension. She consulted a gastroenterologist because of a major weight loss during the past few months, over $10 \mathrm{~kg}$ in 6 months, associated with diarrhea (until 10-12 bowel movements a day). A CT scan was performed and a pancreatic head mass $(15 \times 25 \mathrm{~mm})$ involving the SMV was diagnosed (Fig. 3a), with infracentimetric liver and pulmonary lesions considered aspecific by the local multidisciplinary tumor board. A liver magnetic resonance imaging (MRI) scan was performed, but the lesions were too small to draw a conclusion. Given the contact with the SMV, the tumor was deemed borderline.

Based on the ECOG status of 0 , the patient began systemic treatment with a FOLFIRINOX regimen. Four cycles of chemotherapy were administered. During the treatment, the patient developed fatigue, but global tolerance was acceptable, with an ECOG status remaining between 0 and 1.

After 2 months of treatment, the patient underwent a restaging CT scan (Fig. 3b). It showed tumoral progression and involvement of the right side of the superior mesenteric artery (SMA). The tumor was considered unresectable, and we started palliative chemotherapy with nab-paclitaxel/gemcitabine.

Three cycles of chemotherapy were administered. The patient underwent a new restaging CT scan after the three cycles of chemotherapy, which showed significant tumoral response, with a $29 \%$ regression of the mass and a contact $<180^{\circ}$ with the SMA (Fig. 3c).

According to the regression of the tumor, the patient underwent surgical exploration. The surgeon found no irresectability criteria, so that the patient underwent total duodenopancreatectomy associated with splenectomy. The surgery led to no major complication except for mild malnutrition. As a consequence, enteral nutrition was administered for a few months.

The patient received adjuvant nab-paclitaxel/gemcitabine adjuvant chemotherapy, but only two cycles of chemotherapy were administered due to deterioration of her general health status, with an ECOG status of 3. After the end of chemotherapy, the performance status improved. Within 1 year of regular monitoring with physical examinations, laboratory tests, and CT scans, we have found no sign of tumor recurrence.

\section{Discussion}

The treatment of borderline/locally advanced pancreatic tumors is still controversial. There are different approaches, such as neoadjuvant chemotherapy followed or not by chemoradiation. A meta-analysis published in 2010 [6] showed that approximately onethird of tumor patients initially staged nonresectable would be expected to have resectable 
tumors following neoadjuvant therapy, with a survival comparable to that of initially resectable tumor patients. In the same study, polychemotherapies were superior to monochemotherapies in terms of response and resection rates. Robust data about the role of neoadjuvant chemotherapy in case of locally advanced pancreatic cancer are scarce. In a monocentric study with 22 patients treated with FOLFIRINOX, 5 patients had a R0 resection, with a progression-free survival of 11.7 months [7]. However, this chemotherapy regimen is significantly associated with more hematologic toxicity than gemcitabine alone [4]. The safety profile of nab-paclitaxel/gemcitabine seems to be better [5], even if no direct comparison with FOLFIRINOX was made. Moreover, the nab-paclitaxel plus gemcitabine regimen seemed to be effective after FOLFIRINOX failure in a prospective multicenter cohort study in 57 patients with metastatic pancreatic adenocarcinoma [8].

Another key issue is to determine the eligibility for surgery in borderline/unresectable patients after neoadjuvant treatment. For the 2 described patients, we organized a CT scan after 2 months of chemotherapy. However, a retrospective cohort study showed that the sensitivity and specificity of CT and MRI to detect vascular involvement were only 71 and $58 \%$ after downstaging chemotherapy [9]. Vascular involvement on imaging was often tumor fibrosis rather than viable neoplastic cells. In these 2 cases, the CT scan overestimated the tumors, but the patients could still have a lifesaving R0 resection, which is one of the most significant factor for survival [10].

These 2 cases illustrate that locally advanced pancreatic cancer patients with a good performance status might undergo explorative laparotomy, even after two lines of chemotherapy, as achieving a R0 resection may still be possible in some cases. Moreover, local tumor extension tends to be overestimated by imaging procedures after neoadjuvant chemotherapy.

In these 2 cases, we first prescribed a FOLFIRINOX chemotherapy regimen as neoadjuvant treatment due to its good response rate. However, nab-paclitaxel/gemcitabine can be another valid option, with a better safety profile. A phase II randomized trial comparing nabpaclitaxel/gemcitabine and gemcitabine alone in locally advanced pancreatic cancer patients is ongoing (NCT02043730). Finally, chemoradiotherapy in patients with disease controlled after induction chemotherapy could be a promising treatment, but the available data are conflicting [11, 12]. A phase III study recently began comparing neoadjuvant mFOLFIRINOX with or without preoperative concomitant chemoradiotherapy in patients with borderline resectable pancreatic carcinoma (PANDAS-PRODIGE 44, NCT02676349).

\section{Statement of Ethics}

Informed consent was obtained for this case report.

\section{Disclosure Statement}

The authors have nothing to disclose. 


\section{Case Reports in Gastroenterology}

(C) 2017 The Author(s). Published by S. Karger AG, Basel www.karger.com/crg

Hahn et al: Resection of Locally Advanced Pancreatic Neoplasms after Neoadjuvant Chemotherapy with Nab-Paclitaxel and Gemcitabine following FOLFIRINOX Failure

\section{Author Contributions}

A. Lopez and S. Hahn wrote the manuscript. A. Lopez is the article guarantor. A. Ayav critically revised the manuscript. All authors approved the final manuscript.

\section{References}

1 Siegel RL, Miller KD, Jemal A: Cancer statistics, 2016. CA Cancer J Clin 2016;66:7-30.

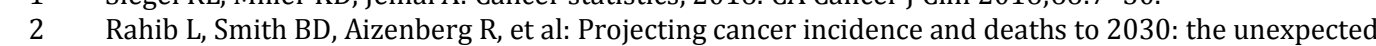
burden of thyroid, liver, and pancreas cancers in the United States. Cancer Res 2014;74:2913-2921.

- G Garrido-Laguna I, Hidalgo M: Pancreatic cancer: from state-of-the-art treatments to promising novel therapies. Nat Rev Clin Oncol 2015;12:319-334.

-4 Conroy T, Desseigne F, Ychou M, et al: FOLFIRINOX versus gemcitabine for metastatic pancreatic cancer. New Engl J Med 2011;364:1817-1825.

5 Von Hoff DD, Ervin T, Arena FP, et al: Increased survival in pancreatic cancer with nab-paclitaxel plus gemcitabine. New Engl J Med 2013;369:1691-1703.

-6 Gillen S, Schuster T, Meyer zum Buschenfelde C, et al: Preoperative/neoadjuvant therapy in pancreatic cancer: a systematic review and meta-analysis of response and resection percentages. PLoS Med 2010;7:e1000267.

7 Faris JE, Blaszkowsky LS, McDermott S, et al: FOLFIRINOX in locally advanced pancreatic cancer: the Massachusetts General Hospital Cancer Center experience. Oncologist 2013;18:543-548.

$\checkmark 8$ Portal A, Pernot S, Tougeron D, et al: Nab-paclitaxel plus gemcitabine for metastatic pancreatic adenocarcinoma after Folfirinox failure: an AGEO prospective multicentre cohort. Br J Cancer 2015;113:989-995.

-9 Donahue TR, Isacoff WH, Hines OJ, et al: Downstaging chemotherapy and alteration in the classic computed tomography/magnetic resonance imaging signs of vascular involvement in patients with pancreaticobiliary malignant tumors: influence on patient selection for surgery. Arch Surg 2011;146: 836-843.

10 Barugola G, Partelli S, Marcucci S, et al: Resectable pancreatic cancer: who really benefits from resection? Ann Surg Oncol 2009;16:3316-3322.

-11 Huguet F, Andre T, Hammel P, et al: Impact of chemoradiotherapy after disease control with chemotherapy in locally advanced pancreatic adenocarcinoma in GERCOR phase II and III studies. J Clin Oncol 2007;25:326-331.

12 Hammel P, Huguet F, van Laethem JL, Goldstein D, Glimelius B, Artru P, et al: Effect of chemoradiotherapy vs chemotherapy on survival in patients with locally advanced pancreatic cancer controlled after 4 months of gemcitabine with or without erlotinib: the LAP07 randomized clinical trial. JAMA 2016;315:1844-1853. 


\section{Case Reports in Gastroenterology}
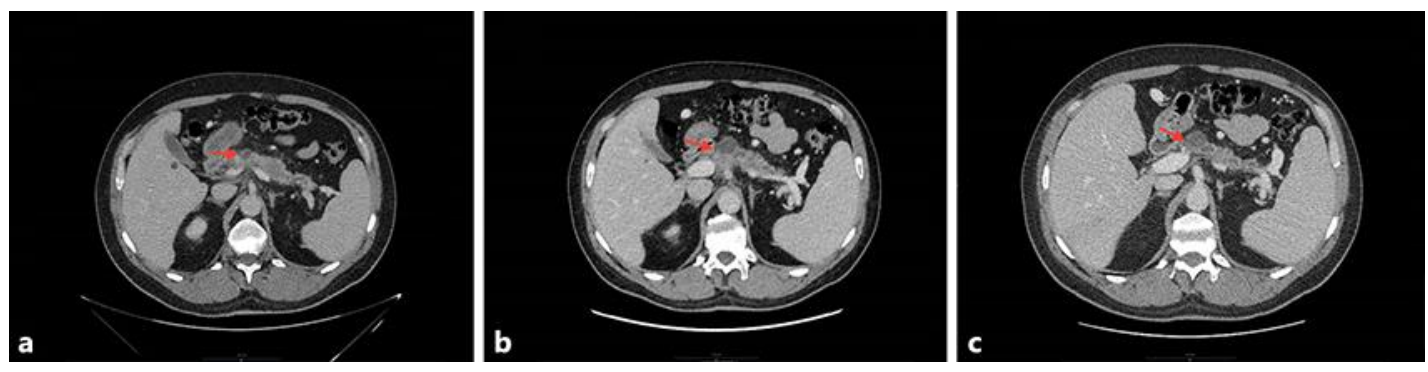

Fig. 1. CT scans of patient 1 at diagnosis (a), after FOLFIRINOX (b), and after nab-paclitaxel/gemcitabine (c).

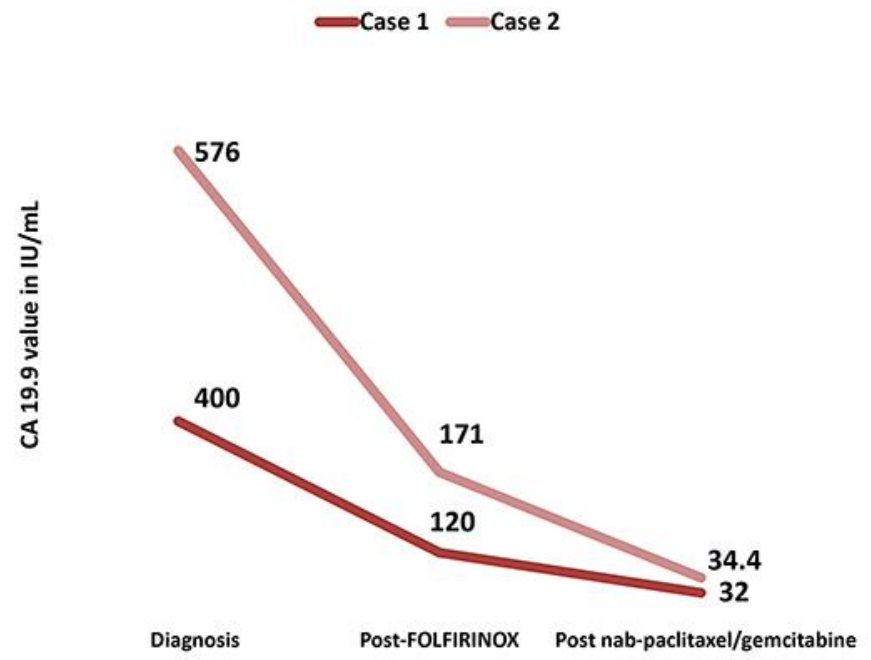

Fig. 2. Evolution of CA19-9 levels in cases 1 and 2.
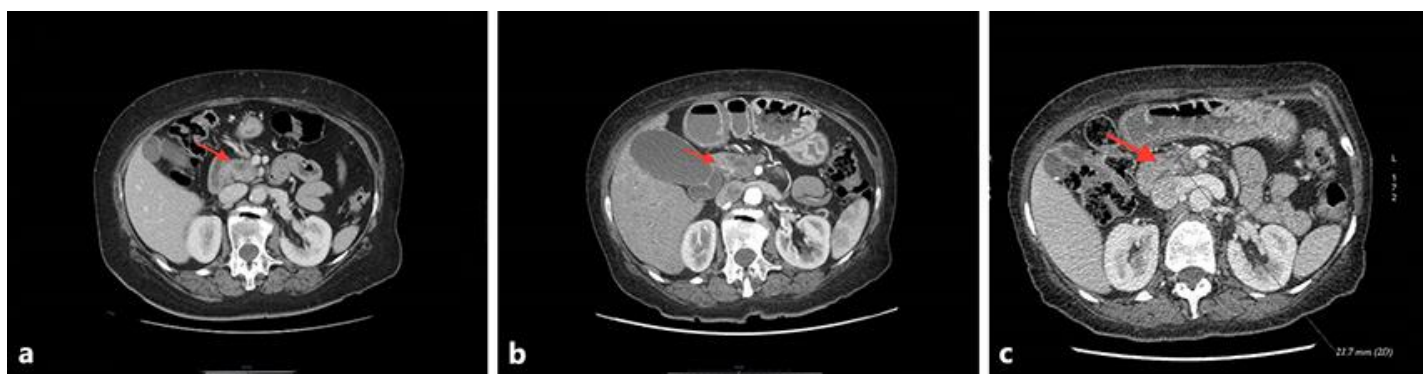

Fig. 3. CT scans of case 2 at diagnosis (a), after FOLFIRINOX (b), and after nab-paclitaxel/gemcitabine (c). 